\section{Validation of the Reflux Disease Questionnaire into Greek}

\author{
Eirini Oikonomidou, ${ }^{1,2}$ \\ Constantinos Mihas, 3 \\ Foteini Anastasiou, 2,4 Christos Lionis \\ ${ }^{1}$ Rural Setting Sindos, Health Centre \\ Diabata; ${ }^{2}$ Clinic of Social and Family \\ Medicine, School of Medicine, University \\ of Crete; ${ }^{3}$ General Hospital-Health Centre \\ of Kimi; ${ }^{4}$ Rural Setting Pyrgos, Health \\ Centre Charaka, Greece
}

\section{Abstract}

Primary care physicians face challenges in diagnosing and managing gastroesophageal reflux disease (GERD). The Reflux Disease Questionnaire (RDQ) meets the standards of validity, reliability, and practicability. This paper reports on the validation of the Greek translation of the RDQ. RDQ is a condition specific instrument. For the validation of the questionnaire, the internal consistency of its items was established using the alpha coefficient of Chronbach. The reproducibility (testretest reliability) was measured by kappa correlation coefficient and the criterion of validity was calculated against the diagnosis of another questionnaire already translated and validated into Greek (IDGP) using kappa correlation coefficient. A factor analysis was also performed. Greek RDQ showed a high overall internal consistency (alpha value: 0.91) for individual comparison. All 8 items regarding heartburn and regurgitation, GERD, had good reproducibility (Cohen's $\kappa$ 0.60-0.79), while the remaining 4 items about dyspepsia had a moderate reproducibility (Cohen's $\kappa=' 0.40$ 0.59) The kappa coefficient for criterion validity for GERD was rather poor $(0.20,95 \%$ CI: $0.04,0.36$ ) and the overall agreement between the results of the RDQ questionnaire and those based on the IDGP questionnaire was $70.5 \%$. Factor analysis indicated 3 factors with Eigenvalue over 1.0, and responsible for $76.91 \%$ of variance. Regurgitation items correlated more strongly with the third component but pain behind sternum and upper stomach pain correlated with the second component. The Greek version of RDQ seems to be a reliable and valid instrument following the pattern of the original questionnaire, and could be used in primary care research in Greece.

\section{Introduction}

Gastroesophageal reflux disease (GERD) is a common condition in Western populations with a prevalence of around $20 \%$ for weekly reflux symptoms that are estimated to make up $4 \%$ of consultations to family physicians. ${ }^{1-3}$ Reviews have highlighted the effect of frequency and severity of GERD symptoms in patients' everyday life. . $^{4}$

There is evidence that primary care physicians face challenges in making an accurate diagnosis of GERD and in managing it effectively. ${ }^{7}$ Heartburn, when present as the predominant symptom, may be the sole basis for the diagnosis of GERD while the imprecise definitions of GERD symptoms, and the overlap between reflux and other upper abdominal symptoms, challenge the diagnostic efficacy of primary care physicians. ${ }^{8}$ What is more, endoscopy is not a substitute for careful evaluation of symptoms since most patients with troublesome reflux symptoms do not have any evidence of endoscopic esophagitis. ${ }^{9}$ Although the 24-h ambulatory $\mathrm{pH}$ monitoring was initially proposed as gold standard, it is not sufficiently sensitive; furthermore, it is expensive, invasive and technically demanding, particularly in the primary care setting. ${ }^{10}$ As an alternative, a brief treatment trial with a proton pump inhibitor may be the most sensitive and specific diagnostic modality. ${ }^{11}$ However, studies on the diagnostic accuracy of the test raise certain concerns.

A European study of primary care patients with GERD indicates that a structured questionnaire could promote better communication and patient management in primary care. ${ }^{12,13}$ Several questionnaires have been developed for the assessment of GERD symptoms in clinical practice or for the general population. ${ }^{12-16}$ The criteria of an ideal evaluative GERD symptom assessment tool are: sensitivity in GERD patients, covering all symptom dimensions (multidimensional construct), assessment of frequency and intensity of typical and atypical GERD symptoms, practical and economical, easy to understand, responsive over short time intervals, use as a patient self-assessment tool, amenable to daily use, psychometrically validated, and being translated into many languages with cross-cultural adaptation. ${ }^{17}$ So far, 14 instruments have been developed to assess GERD symptoms and these can potentially be used to evaluate treatment response during a therapeutic trial in GERD patients. ${ }^{18}$

The Reflux Disease Questionnaire (RDQ), developed by Shaw et al. meets the standards of validity, reliability and responsiveness but also these of practicability and feasibility. ${ }^{16,19-21}$ An observational study in primary care in Greece has focused on the psychometric quali-
Correspondence: Eirini Oikonomidou, Rural Station of Sindos, Health Center of Diabata, 9 I.Kapodistria street Pylaia, Zip Code 55535, Thessaloniki, Greece.

Tel. +30.2310 .326546 - Fax +30.2310 .799697$

E-mail: renaoikonomidou@yahoo.gr

Key words: gastroesophageal reflux disease, questionnaire, Greece.

Acknowledgements: we would like to thank the patients who participated and spent their time to help this research. We would also like to thank in particular Mrs Androniki Glystra, Mrs Sofia Dimopoulou and Mrs Ioanna Tsiligianni for their contribution to the data collection.

Contributions: E0 collected data, draft the manuscript; CM analyzed the statistical data and reviewed the manuscript; FA, collected data, contributed to the study design and participated in the development of the manuscript; CL conceived the initial idea, contributed to the study development, analysis and reporting, reviewed the analysis and interpretation of data, corrected the first draft, co-designed the contents of the manuscript.

Conflict of interests: the authors report no conflict of interests.

Received for publication: 10 February 2012.

Revision received: 26 July 2012.

Accepted for publication: 11 September 2012.

This work is licensed under a Creative Commons Attribution NonCommercial 3.0 License (CC BYNC 3.0).

(C) Copyright E. Oikonomidou et al., 2012

Licensee PAGEPress, Italy

Gastroenterology Insights 2012; 4:e20

doi:10.4081/gi.2012.e20

ties of the Greek RDQ and its potential use in research for clinical practice in primary care. With this in view, this paper reports on the validity of the RDQ in the Greek primary care setting.

\section{Materials and Methods}

\section{Questionnaire}

The RDQ is a condition-specific instrument consisting of 12 questions. Half of the questions are related to the frequency of the symptoms and half to their severity, recording GERD symptoms during the previous week. Symptoms' frequency and severity are measured on 6-point scales (from no occurrence to daily/severe). Patients are asked about the following six symptoms: burning behind breastbone, pain behind breastbone, upper stomach 
burning, upper stomach pain, acid taste in mouth, and movement of materials. The RDQ questionnaire was translated into Greek by a research group from the AstraZeneca GR pharmaceutical company. The authors requested permission from the holders of the rights of the RDQ to validate the questionnaire and adopt it to the Greek conditions.

\section{Setting, sampling and target popu- lation}

The psychometric properties of the Greek version of the RDQ were explored by the administration of the questionnaire to patients with upper gastrointestinal symptoms, seeking primary care in five rural practices serving primary care in Crete and Northern Greece. Eligible patients were recruited for ten consecutive working days. The inclusion criteria were: age over 18 years, absence of alarm symptoms or history of cancer and inflammatory bowel disease. All participants were residents of the practices' area of responsibility. Power analysis showed that in order to discover significant differences higher than $10 \%$ between the responses, the sample size should be 172 individuals, achieving statistical power of $75 \%$ at a significance level of 0.05

\section{Measurements}

Reliability was assessed through internal consistency and test-retest reliability. ${ }^{22-24}$

Internal consistency was determined by checking the components of a questionnaire against each other, using Chronbach's alpha. ${ }^{25,26} \mathrm{~A}$ minimum value of 0.70 for group and 0.90 for individual comparisons is generally desirable. ${ }^{27,28}$

Reproducibility (test-retest reliability) is a significant measure of association for determining the stability of the questionnaire's results over time because it corrects the lack of independence between measurement intervals. ${ }^{23}$ The Cohen's kappa coefficient was also estimated. ${ }^{24}$

Criterion validity refers to the extent to which the instrument correlates with a gold standard. ${ }^{28}$ In order to define the criterion validity of the questionnaire, the diagnoses of the already validated questionnaire, Identification of Dyspepsia in General Population (IDGP), were used as a gold standard to the outcome of the RDQ questionnaire. ${ }^{29}$ The IDGP is the only questionnaire translated and validated which is available in Greek, suitable for the general population and general practice. Kappa analysis was used in order to assess agreement between the diagnoses (GERD, dyspepsia). The diagnosis of GERD using the RDQ was made by the positive response to one of the items $1 \mathrm{a}, 1 \mathrm{~b}, 2 \mathrm{a}$ and $2 \mathrm{~b}$ (heartburn) and/or to one of the items 1e, 1f, $2 \mathrm{e}$ and $2 \mathrm{f}$ (regurgitation of any frequency).
Dyspepsia was diagnosed when there was at least one positive answer to items 1c, 1d, 2c and $2 \mathrm{~d}$. A factor analysis was performed in order to identify the separate factors, which make-up this questionnaire and highlight the way the items group together. ${ }^{30}$ The factor structure was studied by Principal Component Analysis using Varimax with Keiser Normalization as Rotation Method. Both of the Kaiser criteria for applicability were fulfilled. ${ }^{31}$ An analysis of the patients' symptoms as described in the RDQ questionnaire (items 1a, 1b, 1c, 1d, 1e, 1f, 2a, 2b, 2c, 2d, 2e, 2f) was performed and a factor was considered as important if its Eigenvalue value exceeded 1.0. A Bartlett's test of sphericity with $\mathrm{P}<0.05$ and a Kaiser-Meyer-Olkin (KMO) measure of sampling adequacy of 0.734 were used in this factor analysis (Figure 1).

\section{Ethics}

The Scientific and Ethics Committee of the University Hospital of Heraklion, Crete, approved the observational study (protocol $n$. 11873 - 25/10/2006) that aimed to identify the applicability of RDQ in Greek primary care. All participants were informed of the purpose of the study and the confidentiality and anonymity of the process and gave their written consent.

\section{Results}

\section{Sample characteristics}

Patients' demographic and clinical characteristics are presented in Table 1. The final sample consisted of 160 individuals. Twelve individuals who initially entered the study left it early, reducing the statistical power to $72 \%$.

\section{Questionnaire characteristics}

Using the IDGP as the gold standard, the RDQ questionnaire had the following characteristics. For GERD: sensitivity $88.1 \%$, specificity $29.8 \%$, positive predictive value (PPV) $74.4 \%$, negative predictive value (NPV) $51.9 \%$. For dyspepsia: sensitivity $50.0 \%$, specificity 25.7\%, PPV 5.3\%, NPV 86.0\%.

Table 1. Demographic characteristics of the sample.

\begin{tabular}{lccc} 
& Men & Women & Total \\
Age in years* & $64.93 / 13.02$ & $60.36 / 12.60$ & $61.74 / 12.86$ \\
Body mass index $\left(\mathrm{Kg} / \mathrm{m}^{2}\right)^{*}$ & $32.71 / 39.51$ & $28.67 / 4.61$ & $29.98 / 22.76$ \\
\hline Education level & & & \\
None & $2(4 \%)$ & $9(8 \%)$ & $11(7 \%)$ \\
Elementary & $49(96 \%)$ & $97(92 \%)$ & $146(93 \%)$ \\
Married & $50(100 \%)$ & $105(100 \%)$ & $155(100 \%)$ \\
\hline
\end{tabular}

*Mean/standard deviation.
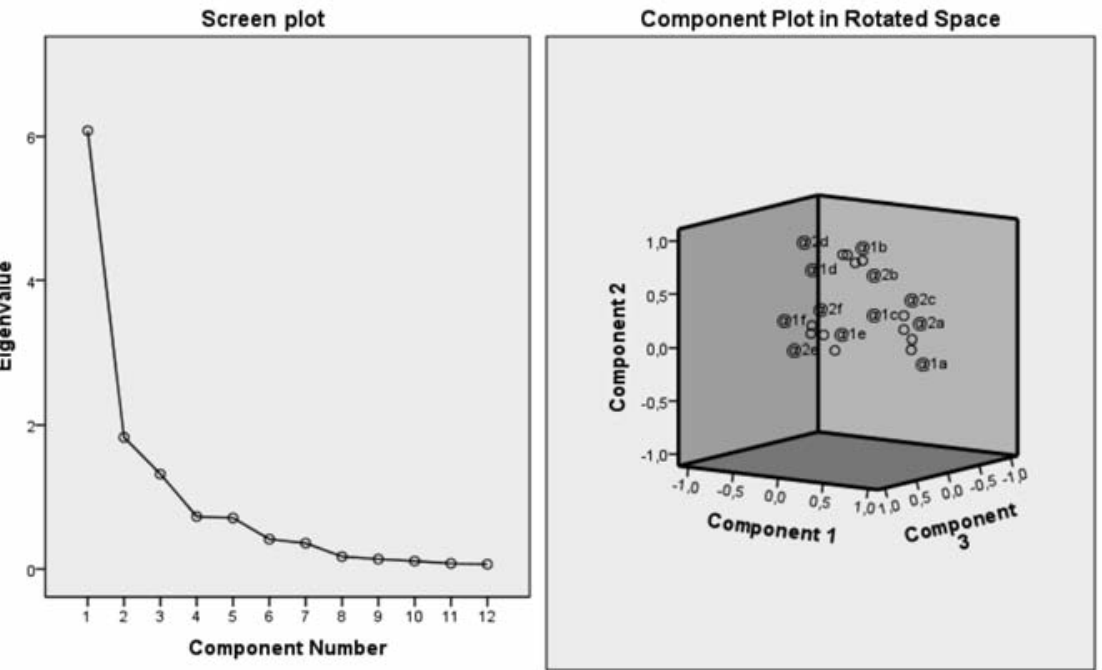

Figure 1. Screen plot and component plot in rotated space. 


\section{Reliability}

The RDQ questionnaire showed a high overall internal consistency (alpha value 0.91) for individual comparison. Each diagnostic group also showed acceptable alpha values: 0.82 for heartburn, 0.88 for regurgitation and 0.83 for dyspepsia. All 8 items regarding heartburn and regurgitation (GERD) had good reproducibility (Cohen's kappa coefficient 0.60-0.79), while the remaining 4 items about dyspepsia had a moderate reproducibility (Cohen's kappa coefficient $0.40-0.59) .{ }^{23}$ Results are shown in Table 2.

\section{Validity}

The kappa coefficient for criterion validity for GERD was quite fair [0.20, 95\% confidence interval (CI): 0.04, 0.36] and the overall agreement between the results of the RDQ questionnaire and those based on the IDGP questionnaire was $70.5 \%$. Regarding dyspepsia, and as expected, the kappa coefficient was poor $(-0.05,95 \%$ CI: $-0.12,0.02)$ and the overall agreement between the results of the $\mathrm{RDQ}$ questionnaire and those based on the IDGP questionnaire was $27.5 \%$.

The performed factor analysis indicated 3 factors with eigenvalue over 1.0. Those factors were responsible for $76.9 \%$ of variance and rotation, and they converged in 5 iterations (Table 3). While regurgitation items correlated more strongly with the third component, pain behind sternum and upper stomach pain correlated with the second component.

\section{Discussion}

\section{Main findings}

The RDQ has been found to be reliable, valid, responsive and above all practical. $16,31-33$ Also, the RDQ was specially designed for use in the primary care setting.

An important feature of the RDQ is the fact that symptoms are described in a figurative, non-technical manner. Previous studies have shown that patients are often confused by terms such as heartburn. ${ }^{12}$ Because of this, the original developers of the RDQ conducted cognitive interviews with patients in order to clarify the obscure questions. Thus, instead of heartburn or regurgitation, researchers used word pictures such as burning behind the breastbone or acid taste in mouth. This characteristic has been retained in the Greek translation. The Greek version of the RDQ showed a high overall internal consistency. The diagnosis of GERD in particular, showed good reproducibility. The criterion validity concerning overall agreement between RDQ and IDGP was rather poor for the diagnosis of GERD and poor for the diagnosis of dyspepsia. The poor agreement could be attributed to the fact that the
RDQ was designed in order to identify GERD rather than exclude dyspepsia, whereas IDGP was designed to identify dyspepsia. Even though we have tried to eliminate this difference by using only those IDGP questions that could lead to a diagnosis of GERD, we are not sure whether the use of another gold standard would have led to different results.

The Greek RDQ has demonstrated high reliability in assessing heartburn and regurgitation. The questions referring to dyspeptic complaints are problematic in the Greek translation, and this was experienced from the Italian and German translations, too. ${ }^{34,35}$ Nevertheless, in their validation Shaw et al. ${ }^{16}$ decided not to delete the dyspepsia-related questions from the RDQ, not only because of their good psychometric results overall but also for the typically overlapping nature of reflux and dyspepsia complaints. In Rome III criteria, GERD is also taken into consideration when dyspeptic patients present symptoms of acid regurgitation or heartburn. ${ }^{36}$
Strengths, limitations and general discussion about the applicability of reflux disease questionnaire

As in usual clinical practice, the diagnosis of GERD was made on the basis of patients' symptoms. However, a comparison using other parameters, such as 24-h ambulatory pH monitoring or upper endoscopy could have been more accurate. We intended to use endoscopy as a gold standard but this was not possible in our study population due to the low compliance of patients; this problem has been analyzed elsewhere. ${ }^{37}$ Consequently, our best approach was considered to be the use of the IDGP questionnaire as being the closest to the reality of Greek primary health care. Future research should rely on more accurate diagnostic tools, and probably another comparison would be preferable for the estimation of RDQ criterion validity. Another characteristic of the Greek validation is that it was performed on people aged over 60 years old, with a low level of edu-

Table 2. Reflux Disease Questionnaire: reproducibility (test- retest reliability).

\begin{tabular}{lccc} 
Symptom & Item & $\mathrm{K}^{*}$ & $\mathrm{P}$ \\
Heartburn & la & 0.753 & $<0.001$ \\
& $1 \mathrm{~b}$ & 0.690 & $<0.001$ \\
& $2 \mathrm{a}$ & 0.616 & $<0.001$ \\
$2 \mathrm{~b}$ & 0.779 & $<0.001$ \\
Regurgitation & $1 \mathrm{e}$ & 0.651 & $<0.001$ \\
& $1 \mathrm{f}$ & 0.627 & $<0.001$ \\
& $2 \mathrm{e}$ & 0.704 & $<0.001$ \\
Dyspepsia & 2f & 0.655 & $<0.001$ \\
& $1 \mathrm{c}$ & 0.531 & $<0.001$ \\
& $1 \mathrm{~d}$ & 0.568 & $<0.001$ \\
& $2 \mathrm{c}$ & 0.515 & $<0.001$ \\
& $2 \mathrm{~d}$ & 0.548 & $<0.001$ \\
\hline
\end{tabular}

${ }^{*}$ Cohen's kappa coefficient.

Table 3. Factor analysis for Reflux Disease Questionnaire items: rotated component matrix for 3 factors.

\begin{tabular}{lccc} 
& & Component & \\
Item-symptom & 1 & 2 & 3 \\
Heartburn & & & \\
la. Burning behind sternum (frequency) & $0.884^{*}$ & 0.061 & 0.257 \\
1b. Pain behind sternum (frequency) & 0.219 & $0.797^{*}$ & 0.199 \\
2a. Burning behind sternum (severity) & $0.865^{*}$ & 0.146 & 0.218 \\
2b. Pain behind sternum (severity) & 0.273 & $0.817^{*}$ & 0.159 \\
Regurgitation & & & \\
le. Acid taste (frequency) & 0.383 & 0.077 & $0.757^{*}$ \\
1f. Movement of materials (frequency) & 0.162 & 0.215 & $0.823^{*}$ \\
2e. Acid taste (severity) & 0.272 & 0.207 & $0.776^{*}$ \\
2f. Movement of materials (severity) & 0.167 & 0.290 & $0.814^{*}$ \\
\hline Dyspepsia & & & \\
1c. Upper stomach burning (frequency) & $0.801^{*}$ & 0.235 & 0.257 \\
1d. Upper stomach pain (frequency) & 0.072 & $0.858^{*}$ & 0.187 \\
2c. Upper stomach burning (severity) & $0.767^{*}$ & 0.356 & 0.208 \\
2d. Upper stomach pain (severity) & 0.134 & $0.864^{*}$ & 0.203 \\
\hline * Highest coefficients. & &
\end{tabular}


cation and this could be considered to be a critical drawback its use in the general population. Nevertheless, it is estimated by country statistics that $60 \%$ of the patients visiting primary care practices in rural areas of Greece are over 60 years of age and have a low level of education. Consequently, the common educational base of the subjects who participated in the research contributes to the strength of the study. The finding that RDQ showed substantial reproducibility is a further advantage of this study. However, its statistical power was not the preferred one ( $72 \%$ instead of $75 \%)$, mainly due to some participants leaving the study.

The RDQ had already been translated into Greek by a research group from Astra-Zeneca. Since there is no literature referring to the translation of RDQ in the Greek language, issues such as the cultural adaptation of the questionnaire cannot be guaranteed by the translating team.

RDQ also focuses on heartburn and regurgitation, and although these are the most typical and prevalent GERD symptoms, there are other symptoms, such as hoarseness or chronic cough, which are not included. This is a general limitation of the questionnaire and should be considered when it is used without any other diagnostic tool.

\section{Implications of the study}

Implications of the validated questionnaire can be demonstrated on a primary care level. It is anticipated that this will represent a practical instrument for primary care physicians in Greece. It can be applied in daily practice for identifying patients with GERD and could be used in epidemiological studies.

\section{Conclusions}

We have accumulated sufficient evidence to show that the Greek version of the RDQ provides a reproducible, reliable and valid instrument in the primary care setting. In addition, the questionnaire is considered to be a useful tool for clinical research in this context. It could also be appropriate for use in international studies, since its psychometric properties are comparable to other versions validated in several countries.

\section{References}

1. Halpern SD, Ubel PA, Caplan AL. Solidorgan transplantation in HIV-infected patients. N Engl J Med 2002;347:284-7.

2. Talley NJ, Zinsmeister AR, Schleck CD, Melton LJ 3rd. Dyspepsia and dyspepsia subgroups: a population-based study. Gastroenterology 1992;102:1259-68.

3. Locke GR 3rd, Talley NJ, Fett SL, et al. Prevalence and clinical spectrum of gastroesophageal reflux: a population-based study in Olmsted County, Minnesota. Gastroenterology 1997;112:1448-56.

4. Dent J, El-Serag HB, Wallander MA, Johansson S. Epidemiology of gastrooesophageal reflux disease: a systematic review. Gut 2005;54:710-7.

5. Kulig M, Leodolter A, Vieth M, et al. Quality of life in relation to symptoms in patients with gastro-oesophageal reflux disease - an analysis based on the ProGERD initiative. Aliment Pharmacol Ther 2003;18:767-76.

6. Wiklund I, Carlsson J, Vakil N. Gastroesophageal reflux symptoms and wellbeing in a random sample of the general population of a Swedish community. Am J Gastroenterol 2006;101:18-28.

7. Ronkainen J, Aro P, Storskrubb T, et al. Gastro-oesophageal reflux symptoms and health-related quality of life in the adult general population - the Kalixanda study. Aliment Pharmacol Ther 2006;23:1725-33.

8. Kirby CN, Piterman L, Nelson MR, Dent J. Gastro-oesophageal reflux disease Impact of guidelines on GP management. Aust Fam Physician 2008;37:73-7.

9. Castell D0, Holtz A. Gastroesophageal reflux. Don't forget to ask about heartburn. Postgrad Med 1989;86:141-8.

10. Jones RH, Hungin AP, Phillips J, Mills JG. Gastro-oesophageal reflux disease in primary care in Europe: clinical presentation and endoscopic findings. Eur J Gen Pract 1995;1:149-54.

11. Ghillibert G, Demeyere AM, Janssens J, Vantrappen G. How well can quantitative 24-hour intraesophageal pH monitoring distinguish various degrees of reflux disease? Dig Dis Sci 1995;40:1317-24.

12. De Leone A, Tonini M, Dominici P, et al. The proton pump inhibitor test for gastroesophageal reflux disease: optimal cut-off value and duration. Dig Liver Dis 2010;42: 785-90.

13. Carlsson R, Dent J, Bolling-Sternevald E, et al. The usefulness of a structured questionnaire in the assessment of symptomatic gastroesophageal reflux disease. Scand J Gastroenterol 1998;33:1023-9.

14. Locke GR, Talley NJ, Weaver AL, Zinsmeister AR. A new questionnaire for gastroesophageal reflux disease. Mayo Clin Proc 1994;69:539-47.

15. Manterola C, Munoz S, Grande L, Bustos L. Initial validation of a questionnaire for detecting gastroesophageal reflux disease in epidemiological settings. J Clin Epidemiol 2002;55:1041-5.

16. Ofman JJ, Shaw M, Sadik K, et al. Identifying patients with gastroesophageal reflux disease: validation of a practical screening tool. Dig Dis Sci 2002;47:1863-9.

17. Shaw MJ, Talley NJ, Beebe TJ, et al. Initial validation of a diagnostic questionnaire for gastroesophageal reflux disease. Am J Gastroenterol 2001;96:52-7.

18. Stanghellini V, Armstrong D, Monnikes H, Bardhan KD. Systematic Review: Do we need a new gastro-oesophageal reflux disease questionnaire? Aliment Pharmacol Ther 2004;19:1-17.

19. Cao Y, Yan X, Ma XQ, et al. Validation of a survey methodology for gastroesophageal reflux disease in China. BMC Gastroenterol 2008;8:37.

20. Nuevo J, Tafalla M, Zapardiel J. Validation of the reflux disease questionnaire (RDQ) and gastrointestinal impact scale (GIS) in patients with gastroesophageal reflux disease in the Spanish population. Gastroenterol Hepatol 2009;32:264-73.

21. Lwanga SK, Lemeshow S. Two-sample situations. In sample size determination in health studies. Geneva: World Health Organization; 1991. pp. 6-8.

22. Cohen J. Statistical power analysis for the behavioral sciences. 2nd ed. Hillsdale: Lawrence Erlbaum; 1988.

23. Altman DG. Some common problems in medical research. In: Practical statistics for medical research. London: Chapman and Hall; 1997. pp. 396-439.

24. Cronbach LJ. Coefficient alpha and the internal structure of tests. Psychometrika 1951;16:297-334.

25. Scientific Advisory Committee of the Medical Outcomes Trust. Assessing health status and quality-of-life instruments: attributes and review criteria. Qual Life Res 2002;11:193-205.

26. Patrick DL, Wild DJ, Johnson ES, et al. Cross-cultural validation of quality of life measures. In: Orley J, Kuyken W. Quality of life assessment: international perspectives. Berlin-Heidelberg: Springer-Verlag; 1994. pp.19-32

27. Bowling A. The principles of research. In: Research methods in health: investigating and health services. 2nd ed. Philadelphia: University Press, Mainhead; 2002. pp.133162.

28. Stevens J. Applied multivariate statistics for the social sciences. London: Lawrence Erlbaum; 1992.

29. Anastasiou F, Antonakis N, Chaireti G, et al. Identifying dyspepsia in the Greek population: translation and validation of a questionnaire. BMC Public Health 2006; 6:56.

30. Aanen M, Numans M, Weusten B, Smout A. Diagnostic value of the reflux disease questionnaire in general practice. Digestion 2006;74:162-8.

31. Dent J, Vakil N, Jones R, et al. Validation of 
the reflux disease questionnaire for the diagnosis of gastroesophageal reflux disease in primary care. Gut 2007;56:A75.

32. Chinese Gastroesophageal Reflux Disease Study Group. Value of reflux diagnostic questionnaire in the diagnosis of gastroesophageal reflux disease. Chin $\mathrm{J}$ Dig Dis 2004;5:51-5.

33. Pace F, Scarlata P, Casini V, et al. Validation of the reflux disease question- naire for an Italian population of patients with gastroesophageal reflux disease. Eur J Gastroenterol Hepatol 2008;20:187-90.

34. Nocon M, Kulig M, Leodolter A, et al. Validation of the reflux disease questionnaire for a German population. Eur $\mathrm{J}$ Gastroenterol Hepatol 2005;17:229-33.

35. Tack J, Talley NJ, Camilleri M, et al. Functional gastroduodenal disorders. Gastroenterology 2006;130:1466-79.
36. Oikonomidou E, Anastasiou F, Pilpilidis I, et al. Upper gastrointestinal endoscopy for dyspepsia: exploratory study of factors influencing patient compliance in Greece. BMC Gastroenterol 2011;14:11.

37. Shaw M, Dent J, Beebe T, et al. The reflux disease questionnaire: a measure for assessment of treatment response in clinical trials. Health Qual Life Outcomes 2008;6:31. 